\title{
Evaluating Convex Roof Entanglement Measures
}

\author{
Géza Tóth, ${ }^{1,2,3,{ }^{*}}$ Tobias Moroder, ${ }^{4}$ and Otfried Gühne ${ }^{4}$ \\ ${ }^{1}$ Department of Theoretical Physics, University of the Basque Country UPV/EHU, E-48080 Bilbao, Spain \\ ${ }^{2}$ IKERBASQUE, Basque Foundation for Science, E-48011 Bilbao, Spain \\ ${ }^{3}$ Wigner Research Centre for Physics, Hungarian Academy of Sciences, H-1525 Budapest, Hungary \\ ${ }^{4}$ Naturwissenschaftlich-Technische Fakultät, Universität Siegen, Walter-Flex-Straße 3, 57068 Siegen, Germany
}

(Received 27 October 2014; published 21 April 2015)

\begin{abstract}
We show a powerful method to compute entanglement measures based on convex roof constructions. In particular, our method is applicable to measures that, for pure states, can be written as low order polynomials of operator expectation values. We show how to compute the linear entropy of entanglement, the linear entanglement of assistance, and a bound on the dimension of the entanglement for bipartite systems. We discuss how to obtain the convex roof of the three-tangle for three-qubit states. We also show how to calculate the linear entropy of entanglement and the quantum Fisher information based on partial information or device independent information. We demonstrate the usefulness of our method by concrete examples.
\end{abstract}

DOI: 10.1103/PhysRevLett.114.160501

PACS numbers: 03.67.Mn, 03.65.Ud, 42.50.St

Quantum entanglement plays a central role in quantum information science and quantum optics [1]. There are now efficient methods to detect entanglement, that have even been used in many experiments [2]. These mostly answer the yes or no question "Is the quantum state entangled?" or "Is the quantum state genuine multipartite entangled?" After verifying the presence of entanglement, the next step is quantifying it. Calculating measures is becoming increasingly important in experiments in quantum information science [3-5] and it also plays a crucial role in investigations in quantum statistical physics, e.g., in studying phase transitions [6].

Most entanglement measures are based on the convex roof of a quantity on pure states such as the entropy of the reduced state [7-9]. Measures of this type can also be used to classify states according to their membership in some convex sets, for example, based on their Schmidt rank $[10,11]$. They play a central role in quantum information theory, however, in most of the cases they are not computable as there are no efficient ways to calculate convex roofs. Most importantly, the simplest multipartite entanglement measure, the three-tangle for three qubits, cannot be computed for a general state.

Thus, for obtaining entanglement measures in theory and experiments, it would be crucial to find methods to calculate convex roof constructions efficiently, at least for not too large systems. This seems to be a very difficult task since straightforward numerical search means an optimization over an infinite number of convex decompositions of the density matrix. Such an approach will lead

Published by the American Physical Society under the terms of the Creative Commons Attribution 3.0 License. Further distribution of this work must maintain attribution to the author $(s)$ and the published article's title, journal citation, and DOI. to an upper bound on the measure, since a multivariable numerical optimization is not guaranteed to find the global optimum [12]. Upper bounds, however, are often not very useful as the amount of entanglement can be much lower or even zero even if the procedure signals considerable entanglement.

In this Letter, we present a method that produces a series of very good lower bounds on important entanglement measures. Our method has the following characteristics. (i) It is based on semidefinite programming. The series of bounds obtained converge in a controllable way to the true value. Even the first lower bound in the series is nontrivial. (ii) We have a clear physical picture for what states our method yields a nonzero value for the measures. (iii) The set of separable states is used in the optimization procedure. This way we connect calculating convex roofs to the separability problem, which might help to find applications of the separability problem in other areas of physics.

Hence, our method has far reaching consequences in entanglement theory that can also be seen from its many applications. We will demonstrate the use of our method with the example of computing bipartite entanglement measures for bound entangled states, computing the convex roof of the tangle for various three-qubit states, and even quantities outside of quantum information science. Our method can also be used to compute a lower bound from incomplete data of the quantum state or in device independent scenarios [13-16]. This also implies that semidefinite programming, which has been used before [17-19], can now be applied to a large set of so-far unsolved problems.

Convex roof of linear entropy.-For pure states, the linear entropy of entanglement is given as

$$
E_{\text {lin }}(|\Psi\rangle)=S_{\text {lin }}\left[\operatorname{Tr}_{1}(|\Psi\rangle)\right],
$$


where we used the definition of the linear entropy $S_{\text {lin }}(\varrho)=1-\operatorname{Tr}\left(\varrho^{2}\right)$. Hence, the linear entropy of entanglement for pure states equals also $C^{2} / 2$, where $C$ is the concurrence [7], and it is also equal to the $I$ tangle [20]. The definition (1) can be extended to mixed states by a convex roof construction as

$$
E_{\text {lin }}(\varrho)=\min _{\left.\left\{p_{k}, \Psi_{k}\right\rangle\right\}}\left(\sum_{k} p_{k} E_{\operatorname{lin}}\left(\left|\Psi_{k}\right\rangle\right)\right)
$$

where $\left\{p_{k},\left|\Psi_{k}\right\rangle\right\}$ is a decomposition to pure states

$$
\varrho=\sum_{k} p_{k}\left|\Psi_{k}\right\rangle\left\langle\Psi_{k}\right|
$$

It can be shown that $E_{\text {lin }}(\varrho)$ does not increase under local operations and classical communication (LOCC) on average; hence, it is an entanglement monotone [21]. Consequently, $E_{\text {lin }}(\varrho)$ has also been used to characterize entanglement even in the multipartite setting [22].

Next, we will show a method to compute Eq. (2). For this aim, first we write the liner entropy of entanglement as an expectation value of an operator acting on two copies of a bipartite pure state $|\Psi\rangle$ as [23]

$$
E_{\text {lin }}(|\Psi\rangle)=\operatorname{Tr}\left[\mathcal{A}_{\mathrm{AA}^{\prime}} \otimes \mathbb{1}_{B B^{\prime}}(|\Psi\rangle\langle\Psi|)_{A B} \otimes(|\Psi\rangle\langle\Psi|)_{A^{\prime} B^{\prime}}\right] .
$$

Here, $A$ and $B$ denote the parties of the first copy while $A^{\prime}$ and $B^{\prime}$ denote the parties of the second copy. Moreover, the projector to the antisymmetric space is defined as $\mathcal{A}_{\mathrm{AA}^{\prime}}:=(\mathbb{1}-\mathcal{F})_{\mathrm{AA}^{\prime}}, \mathcal{F}$ is the flip operator, and we explicitly wrote out $\mathbb{1}_{B B^{\prime}}$ for clarity [24].

Next, we will consider mixed states. Let us assume that $\left\{\tilde{p}_{k},\left|\tilde{\Psi}_{k}\right\rangle\right\}$ is the decomposition attaining the convex roof. Then, for a state with such a decomposition we obtain

$$
\begin{aligned}
E_{\text {lin }}(\varrho) & =\sum_{k} \tilde{p}_{k} E_{\operatorname{lin}}\left(\left|\tilde{\Psi}_{k}\right\rangle\right) \\
& =\sum_{k} \tilde{p}_{k} \operatorname{Tr}\left(\mathcal{A}_{\mathrm{AA}^{\prime}}\left|\tilde{\Psi}_{k}\right\rangle\left\langle\left.\tilde{\Psi}_{k}\right|^{\otimes 2}\right)\right. \\
& =\operatorname{Tr}\left(\mathcal{A}_{\mathrm{AA}^{\prime}} \omega_{12}\right),
\end{aligned}
$$

where the state on the two-copy space is defined as

$$
\omega_{12}=\sum_{k} \tilde{p}_{k}\left|\tilde{\Psi}_{k}\right\rangle\left\langle\tilde{\Psi}_{k}|\otimes| \tilde{\Psi}_{k}\right\rangle\left\langle\tilde{\Psi}_{k}\right|
$$

The density matrix $\omega_{12}$ has three important properties. It is a mixture of product states, i.e., a separable state [25]. Moreover, all the pure product components are symmetric. Thus, $\omega_{12}$ is supported on the symmetric subspace. In fact, any symmetric separable states can be written in the form (6) [26]. Finally, $\operatorname{Tr}_{2}\left(\omega_{12}\right)=\varrho$.
Hence, we arrive at our first main result.

Observation 1.-The convex roof of the linear entropy can be written as

$$
E_{\text {lin }}(\varrho)=\min _{\omega_{12}} \operatorname{Tr}\left(\mathcal{A}_{\mathrm{AA}^{\prime}} \omega_{12}\right),
$$

s.t. $\omega_{12}$ symmetric, separable, $\omega_{1}=\varrho$,

where $\omega_{1} \equiv \operatorname{Tr}_{2}\left(\omega_{12}\right)$.

Observation 1 connects the separability problem of symmetric bipartite states, i.e., answering the question "Is the state entangled?" mentioned in the introduction, to entanglement quantification. In principle, to obtain a lower bound on $E_{\text {lin }}(\varrho)$, any necessary condition for separability could be used. We will consider the method based on the positivity of partial transpose (PPT) [27] and obtain a lower bound as

$$
\begin{gathered}
E_{\text {lin }}^{(\mathrm{PPT})}(\varrho)=\min _{\omega_{12}} \operatorname{Tr}\left(\mathcal{A}_{\mathrm{AA}^{\prime}} \omega_{12}\right), \\
\text { s.t. } \omega_{12} \text { symmetric, PPT, } \quad \omega_{1}=\varrho .
\end{gathered}
$$

Next, we will demonstrate that our method can be used to quantify the entanglement of states not detected by the PPT condition, called bound entangled states [28-30].

Horodecki state.-We test our method to calculate entanglement measures for the one-parameter family of the $3 \times 3$ bound entangled state $\varrho_{a}^{\mathrm{PH}}$ introduced by Horodecki [29]. We mix the state with white noise according to $\varrho_{a}(p)=p \varrho_{a}^{\mathrm{PH}}+(1-p) \mathbb{1} / 9$ and calculate the entanglement as a function of $a$ and $p$. The results can be seen in Fig. 1. The critical noise for which $E_{\text {lin }}^{(\mathrm{PPT})}(\varrho)=0$ agrees with the calculations of Ref. [24] and Ref. [31]. We note that we made the computer program calculating $E_{\text {lin }}^{(\mathrm{PPT})}(\varrho)$, with other programs used for this publication, publicly available [32]. Other methods for calculating entanglement measures are in Refs. [33,34].

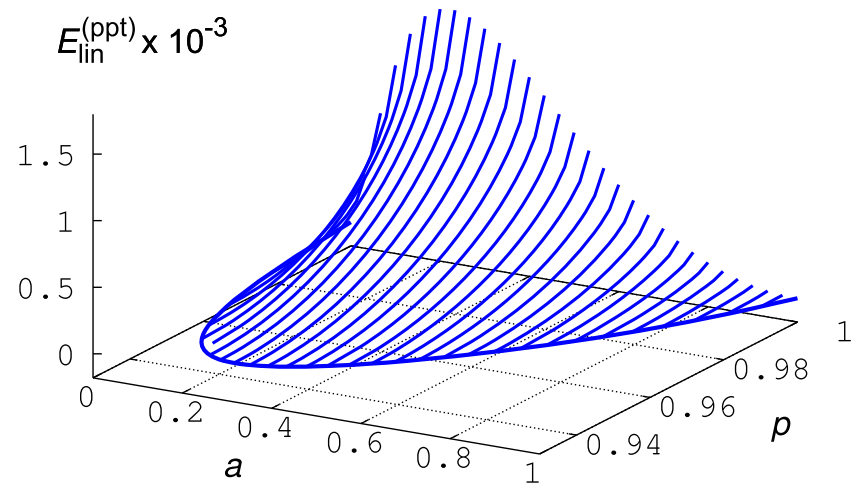

FIG. 1 (color online). Entanglement quantification for the noisy $3 \times 3$ Horodecki bound entangled state $\varrho_{a}(p)$, using $E_{\text {lin }}^{(\mathrm{PPT})}(\varrho)$ defined after observation 1 . We plot $E_{\text {lin }}^{(\mathrm{PPT})}(\varrho)$ as a function of the parameter of the state, $a$, and the weight-parameter $p$. 
It is a surprise that, while the bound relies on the PPT criterion, the method is still able to detect PPT entangled states. In order to obtain more information on what kind of states are detected, we need to know the separability criterion based on symmetric extensions [18]. A given bipartite state $Q_{A B}$ is said to have a $n: m$ symmetric extension if it can be written as the reduced state of a multipartite state $\varrho_{A_{1} \ldots A_{n} B_{1} \ldots B_{m}}$, which is symmetric under $A_{k} \leftrightarrow A_{l}$ and $B_{k} \leftrightarrow B_{l}$ for all $k \neq l$. If we also require that the state is PPT for all bipartitions, then it is a PPT symmetric extension. Separable states have such extensions for arbitrarily large $n$ and $m$, while the lack of such an extension signals the presence of entanglement.

Observation 2.-For all non-PPT states and for all states that do not have a 2:2 symmetric extension we have $E_{\text {lin }}^{(\mathrm{PPT})}(\varrho)>0$. Moreover, for all states having a 2:2 PPT symmetric extension $E_{\text {lin }}^{(\mathrm{PPT})}(\varrho)=0$ holds. The proof can be found in the Supplemental Material [35].

Before we continue let us point out that we can also obtain a lower bound on $E_{\text {lin }}(\varrho)$ if we choose any other entanglement condition, such as the method based on local uncertainty relations [48], the covariance matrix criterion [49], or the computable cross norm or realignment criterion (CCNR) [50]. However, for symmetric states these are all equivalent to the PPT condition [51].

Therefore, to strengthen the bound a stronger criterion must be employed. Here again the method of PPT symmetric extensions can be used [18]. Rather than approximating $\omega_{12}$ by PPT states, we demand that $\omega_{12}$ has an $n: 1$ PPT symmetric extension [52]. In this way we obtain a sequence of lower bounds $E_{\text {lin }}^{(n)}$ with increasing accuracies. The corresponding optimization can similarly be carried out by semidefinite programming. Note that the PPT symmetric extensions converge to the set of separable states in a controlled way [53]. Finally, note also that semidefinite programs not only detect entanglement, but through solving the dual problem, it is possible to find entanglement witnesses [18]. In our case, these witnesses can even bound entanglement measures, as explained in the Supplemental Material [35].

Generalization and further examples.-The previous ideas can straightforwardly be generalized to compute the convex roof of any quantity that can be written as a polynomial of expectation values for pure states as

$$
E(|\Psi\rangle)=\sum_{m=1}^{M} \sum_{n=0}^{N} c_{m n}\left\langle A_{m}\right\rangle^{n},
$$

where $A_{m}$ are operators and $c_{m n}$ are constants (see, e.g., $[54,55])$. It is possible to define an operator $L:=$ $\sum_{m, n} c_{m n} A_{m}^{\otimes n} \otimes \mathbb{1}^{\otimes(N-n)}$, whose expectation value on several copies reproduces Eq. (9). Then, the convex roof of Eq. (9) can be obtained as an optimization over $N$-copy symmetric fully separable states [26]

$$
E(\varrho)=\min _{\omega_{12 . . N}} \operatorname{Tr}\left(L \omega_{12 . . N}\right),
$$

s.t. $\omega_{12 . . N}$ symmetric, fully separable, $\quad \omega_{1}=\varrho$.

Three-tangle.-Our next example is the calculation of the three-tangle, a three-qubit entanglement monotone [56]. For pure states, it has been defined by Coffmann, Kundu, and Wootters [9]. Remarkably, it can be written as a fourthorder polynomial in expectation values [54]. Hence, for mixed states, the tangle can be defined through a convex roof extension, which we can now map to the optimization problem

$$
\tau(\varrho)=\min _{\omega_{1234}} \operatorname{Tr}\left(T \omega_{1234}\right),
$$

s.t. $\omega_{1234}$ symmetric, fully separable, $\omega_{1}=\varrho$,

where $T$ is an operator acting on four copies of the threequbit state [57]. Note that if we know $\tau(\varrho)$, we can decide whether a three-qubit fully entangled state is in the $\mathrm{W}$ or in the GHZ class [10].

The optimization can be carried out for symmetric multiqubit states that are PPT with respect to all bipartitions rather than symmetric separable states, leading to the lower bound $\tau^{(\mathrm{PPT})}$. The results are shown in Fig. 2 for states of the form

$$
\begin{aligned}
\varrho(x, y)= & x\left|\mathrm{GHZ}^{+}\right\rangle\left\langle\mathrm{GHZ}^{+}|+y| \mathrm{GHZ}^{-}\right\rangle\left\langle\mathrm{GHZ}^{-}\right| \\
& +(1-x-y)|\mathrm{W}\rangle\langle\mathrm{W}|,
\end{aligned}
$$

where $\left|\mathrm{GHZ}^{ \pm}\right\rangle=(|000\rangle \pm|111\rangle) / \sqrt{2}$, and $|\mathrm{W}\rangle=(|100\rangle+$ $|010\rangle+|001\rangle) / \sqrt{3}$. Note that a lower bound for the convex roof of the tangle for general states, which is exact for states with certain symmetries, has been developed [58].

As a practical comment, we add that the numerical computation is challenging, but $\tau^{(\mathrm{PPT})}$ can be computed on

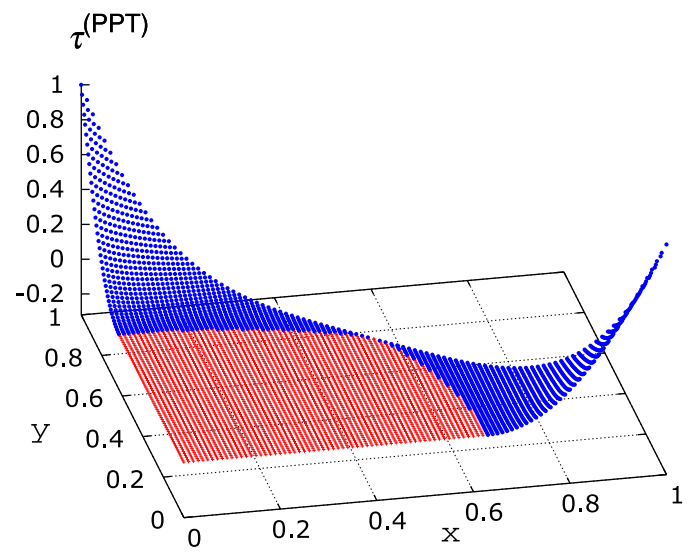

FIG. 2 (color online). Three-tangle of a family of states (12) as a function of the parameters $x$ and $y$. Light color indicates the region where the tangle is zero, darker color indicates a nonzero value. 
a standard laptop with standard free packages for semidefinite programming [59], if the state has some symmetries, or has a rank up to six. Calculations for general three-qubit states of rank eight are realistic with computer clusters and professional packages. Note also that with standard free packages $E_{\text {lin }}^{(\mathrm{PPT})}$ can be computed for general two-qudit states, while $E_{\text {lin }}^{(2)}$ can be obtained for states with a nonmaximal rank. According to our experience, with the current semidefinite solvers, the difference between the two is within the numerical precision.

Schmidt rank.-Let us consider the quantities $R_{r}$ that are nonzero for states with a Schmidt rank larger than $r$. For example, $\quad R_{2}(|\Psi\rangle)=\sum_{i<j} \lambda_{i} \lambda_{j} \equiv 4 S_{\text {lin }}(|\Psi\rangle), \quad R_{3}(|\Psi\rangle)=$ $\sum_{i<j<k} \lambda_{i} \lambda_{j} \lambda_{k}$, where $\lambda_{k}$ are the eigenvalues of the reduced state. The $R_{k}$ quantities are proven to be entanglement monotones [60]. We can calculate the convex roof of $R_{r}$ with our method. Convex roofs for such quantities allow us to bound the dimensionality of the entanglement from below. A powerful bound can be obtained by carrying out the optimization for $r$-qudit symmetric states that are PPT with respect to all bipartitions. An alternative is computing the negativity [61,62]. In particular, $\mathcal{N}(\varrho)-1 / 2>0$ signals that the Schmidt number is larger than 2 . We show that our method outperforms the negativity as a dimension witness in Fig. 3(a) for the family of states

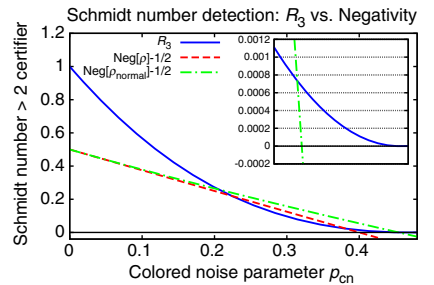

(a)

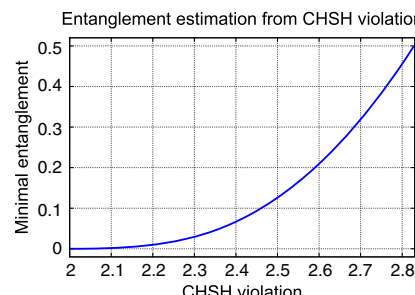

(c)

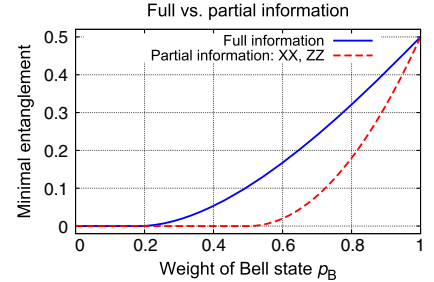

(b)

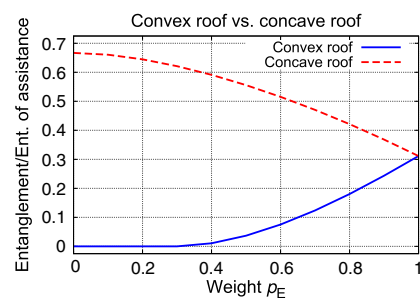

(d)
FIG. 3 (color online). (a) Schmidt-number witness vs negativity for a state of the type (13) as a function of $p_{\mathrm{cn}}$. As the inset shows, even when we consider negativity of the normal form, obtained through stochastic local operations and classical communications (SLOCC) such that all local matrices are fully mixed [63], our numerical method is superior. (b) $E_{\text {lin }}^{(\mathrm{PPT})}(\varrho)$, given in Eq. (8), based on partial information for the state (14). (c) Estimation of $E_{\text {lin }}^{(\mathrm{PPT})}(\varrho)$ as a function of the violation of the CHSH inequality. (d) $E_{\operatorname{lin}}^{(\mathrm{PPT})}(\varrho)$ and the corresponding bound for the entanglement of assistance (defined with the linear entropy) for a state of the type (15) as a function of $p$.

$$
\varrho_{\mathrm{S}}\left(p_{\mathrm{cn}}\right)=\left(1-p_{\mathrm{cn}}\right)\left|\Psi_{\mathrm{S}}\right\rangle\left\langle\Psi_{\mathrm{S}}\right|+p_{\mathrm{cn}} \varrho_{\mathrm{cn}},
$$

with $\left|\Psi_{\mathrm{S}}\right\rangle=(|00\rangle+|11\rangle+|22\rangle) / \sqrt{3}$ and colored noise $\varrho_{\text {cn }}=\mathbb{1}_{2} \otimes \mathbb{1}_{2} / 4$ with $\mathbb{1}_{2}=(|0\rangle\langle 0|+| 1\rangle\langle 1|)$.

We add that we checked several random $3 \times 3$ edge states to test the conjecture of Sanpera, Bruß, and Lewenstein claiming that all bound entangled states in such systems have a Schmidt rank 2, and did not find a counterexample [11].

Evaluation of entanglement measures based on incomplete information.-Experimentally, it is very important that entanglement measures can be evaluated based on incomplete knowledge on the quantum state. There are efficient methods to bound entanglement measures based on an operator expectation value from below [3-5]. The current method can be adapted straightforwardly to the partial information case by replacing the condition $\omega_{1}=\varrho$ with the set of linear constraints $\operatorname{Tr}\left(\omega_{1} O_{i}\right)=v_{i}$, where $O_{i}$ are the measured observables and $v_{i}$ are the corresponding expectation values. As an example, see Fig. 3(b), where the entanglement is bounded from below based on complete information and based on $\left\langle\sigma_{x} \otimes \sigma_{x}\right\rangle$ and $\left\langle\sigma_{z} \otimes \sigma_{z}\right\rangle$ measurements for the state

$$
\varrho_{B}(p)=p_{B}\left|\Phi_{3 \times 3}^{+}\right\rangle\left\langle\Phi_{3 \times 3}^{+}\right|+\left(1-p_{B}\right) \frac{1}{9} \mathbb{1},
$$

where $\left|\Phi_{3 \times 3}^{+}\right\rangle$is a two-qubit Bell state $(|00\rangle+|11\rangle) / \sqrt{2}$ embedded in the $3 \times 3$ system and $\sigma_{l} \otimes \sigma_{l}$ acts on this two-qubit system.

Device independent scenario.-The amount of entanglement can be bounded exclusively from the observed data but independent of the quantum description. Depending whether only one or both sides are untrusted one distinguishes between a steering-type or a Bell-type scenario. The necessary steps to lift the method using only partial information to such device independent scenarios employs the translation idea highlighted in Ref. [15] and is explained in more detail in the Supplemental Material [35]. As an example, in Fig. 3(c) we plot a lower bound on the linear entropy of entanglement given as a function of the violation of the CHSH Bell inequality [1].

Concave roof.-Besides convex roofs, concave roofs can also be computed. For example, if in Eq. (2) a concave roof is used instead of a convex roof, then we compute the linear entanglement of assistance [64], which is the maximal entanglement available if the mixed state is given as a purification to us, and a third party which holds the ancilla needed for the purification is assisting us. In this case, in our method minimum must be replaced by maximum. In this way, we obtain a converging series of upper bounds on the entanglement of assistance. The results are shown in Fig. 3(d) for the family of $3 \times 3$ states

$$
\varrho_{E}\left(p_{E}\right)=p_{E}\left|\Psi_{E}\right\rangle\left\langle\Psi_{E}\right|+\left(1-p_{E}\right) \frac{1}{9} \mathbb{1},
$$


where $\left.\left|\Psi_{E}\right\rangle=\epsilon|00\rangle+\epsilon|11\rangle+\sqrt{1-2 \epsilon^{2}} 22\right\rangle$ and $\epsilon=0.3$. As a reference, the linear entropy of entanglement is also shown for the same state.

Conclusions.-We have shown a general framework for calculating convex roof-based entanglement measures. We demonstrated its use in calculating the entanglement for bipartite systems, as well as the three-tangle for three qubits. We discussed several other quantities for which it can be applied. In the future, we would like to explore further possibilities of using our algorithm to compute convex roofs, in calculating the linear Holevo capacity $[65,66]$, the quantum Fisher information based on incomplete information [67], or the convex or concave roofs of sums of several variances, as outlined in the Supplemental Material [35]. The programs used for this Letter are publicly available [32], which makes it possible to use our method immediately in experiments.

We thank T. Bastin, C. Eltschka, M. Kús, J. Siewert, and M. Tiersch for stimulating discussions. We thank the support of the EU (ERC Starting Grant No. GEDENTQOPT, No. CHIST-ERA QUASAR, Marie Curie CIG 293993/ ENFOQI), the MINECO (Project No. FIS2012-36673C03-03), the Basque Government (Project No. IT472010), the OTKA (Contract No. K83858), the UPV/EHU program UFI 11/55, the FQXi Fund (Silicon Valley Community Foundation), and the DFG.

*toth@alumni.nd.edu; http://www.gtoth.eu

[1] R. Horodecki, P. Horodecki, M. Horodecki, and K. Horodecki, Rev. Mod. Phys. 81, 865 (2009); O. Gühne and G. Tóth, Phys. Rep. 474, 1 (2009).

[2] J.-W.Pan, D. Bouwmeester, M. Daniell, H. Weinfurter, and A. Zeilinger, Nature (London) 403, 515 (2000); M. Bourennane, M. Eibl, C. Kurtsiefer, S. Gaertner, H. Weinfurter, O. Gühne, P. Hyllus, D. Bruß, M. Lewenstein, and A. Sanpera, Phys. Rev. Lett. 92, 087902 (2004); W. Wieczorek, R. Krischek, N. Kiesel, P. Michelberger, G. Tóth, and H. Weinfurter, Phys. Rev. Lett. 103, 020504 (2009).

[3] O. Gühne, M. Reimpell, and R. F. Werner, Phys. Rev. Lett. 98, 110502 (2007); Phys. Rev. A 77, 052317 (2008).

[4] J. Eisert, F. G. S. L. Brandao, and K. M. R. Audenaert, New J. Phys. 9, 46 (2007).

[5] H. Wunderlich and M. B. Plenio, J. Mod. Opt. 56, 2100 (2009).

[6] A. Osterloh, L. Amico, G. Falci, and R. Fazio, Nature (London) 416, 608 (2002); M. Cramer, A. Bernard, N. Fabbri, L. Fallani, C. Fort, S. Rosi, F. Caruso, M. Inguscio, and M. B. Plenio, Nat. Commun. 4, 2161 (2013).

[7] W. K. Wootters, Phys. Rev. Lett. 80, 2245 (1998).

[8] T.-C. Wei and P. M. Goldbart, Phys. Rev. A 68, 042307 (2003).

[9] V. Coffman, J. Kundu, and W. K. Wootters, Phys. Rev. A 61, 052306 (2000).

[10] A. Acín, D. Bruß, M. Lewenstein, and A. Sanpera, Phys. Rev. Lett. 87, 040401 (2001).
[11] A. Sanpera, D. Bruß, and M. Lewenstein, Phys. Rev. A 63, 050301 (2001).

[12] B. Röthlisberger, J. Lehmann, and D. Loss, Phys. Rev. A 80, 042301 (2009).

[13] F. Verstraete and M. M. Wolf, Phys. Rev. Lett. 89, 170401 (2002).

[14] Y.-C. Liang, T. Vértesi, and N. Brunner, Phys. Rev. A 83, 022108 (2011).

[15] T. Moroder, J. D. Bancal, Y. C. Liang, M. Hofmann, and O. Gühne, Phys. Rev. Lett. 111, 030501 (2013).

[16] M. Pusey, Phys. Rev. A 88, 032313 (2013)

[17] B. M. Terhal, A. C. Doherty, and D. Schwab, Phys. Rev. Lett. 90, 157903 (2003).

[18] A. C. Doherty, P. A. Parrilo, and F. M. Spedalieri, Phys. Rev. A 69, 022308 (2004); 71, 032333 (2005).

[19] D.-L. Deng, J.-L. Chen, and Z.-S. Zhou, arXiv: 0909.2919v2.

[20] P. Rungta, V. Buzek, C. M. Caves, M. Hillery, and G. J. Milburn, Phys. Rev. A 64, 042315 (2001).

[21] P. Rungta and C. M. Caves, Phys. Rev. A 67, 012307 (2003); G. Vidal, J. Mod. Opt. 47, 355 (2000).

[22] M. Huber and J. I. de Vicente, Phys. Rev. Lett. 110, 030501 (2013); M. Huber, M. Perarnau-Llobet, and J. I. de Vicente, Phys. Rev. A 88, 042328 (2013).

[23] P. Horodecki, Phys. Rev. A 68, 052101 (2003).

[24] F. Mintert, M. Kus, and A. Buchleitner, Phys. Rev. Lett. 95, 260502 (2005).

[25] R. F. Werner, Phys. Rev. A 40, 4277 (1989).

[26] Every symmetric separable state of $N$ particles can be written as $\sum_{k} q_{k}\left|\Phi_{k}\right\rangle\left\langle\left.\Phi_{k}\right|^{\otimes N}, \sum_{k} q_{k}=1\right.$, and $q_{k}>0$. See J. K. Korbicz, J. I. Cirac, and M. Lewenstein, Phys. Rev. Lett. 95, 120502 (2005).

[27] A. Peres, Phys. Rev. Lett. 77, 1413 (1996); M. Horodecki, P. Horodecki, and R. Horodecki, Phys. Lett. A 223, 1 (1996).

[28] M. Horodecki, P. Horodecki, and R. Horodecki, Phys. Rev. Lett. 80, 5239 (1998); for a review, see P. Horodecki in Lectures on Quantum Information, edited by D. Bruß and G. Leuchs (Wiley-VCH, Berlin, 2006).

[29] P. Horodecki, Phys. Lett. A 232, 333 (1997).

[30] K. Horodecki, M. Horodecki, P. Horodecki, and J. Oppenheim, Phys. Rev. Lett. 94, 160502 (2005); A. Acín, J. I. Cirac, and L. Masanes, Phys. Rev. Lett. 92, 107903 (2004); G. Tóth, C. Knapp, O. Gühne, and H. J. Briegel, Phys. Rev. Lett. 99, 250405 (2007).

[31] Z.-H. Chen, Z.-H. Ma, O. Gühne, and S. Severini, Phys. Rev. Lett. 109, 200503 (2012).

[32] The CoRoNA package can be downloaded at http://www .mathworks.com/matlabcentral/fileexchange/47823-corona -convex-roof-numerical-analysis.

[33] K. Chen, S. Albeverio, and S.-M. Fei, Phys. Rev. Lett. 95, 040504 (2005); J. I. de Vicente, Phys. Rev. A 75, 052320 (2007); 77, 039903(E) (2008); O. Gittsovich and O. Gühne, Phys. Rev. A 81, 032333 (2010).

[34] E. Gerjuoy, Phys. Rev. A 67, 052308 (2003); Y.-C. Ou, H. Fan, and S.-M. Fei, Phys. Rev. A 78, 012311 (2008); M. Li, S.-M. Fei, and Z.-X. Wang, J. Phys. A 42, 145303 (2009).

[35] See Supplemental Material at http://link.aps.org/ supplemental/10.1103/PhysRevLett.114.160501, which includes Refs. [36-47]. 
[36] D. A. Meyer and N. R. Wallach, J. Math. Phys. (N.Y.) 43, 4273 (2002).

[37] A. J. Scott, Phys. Rev. A 69, 052330 (2004).

[38] G. Tóth, Phys. Rev. A 85, 022322 (2012); P. Hyllus, W. Laskowski, R. Krischek, C. Schwemmer, W. Wieczorek, H. Weinfurter, L. Pezzé, and A. Smerzi, Phys. Rev. A 85, 022321 (2012).

[39] G. Tóth, C. Knapp, O. Gühne, and H. J. Briegel, Phys. Rev. Lett. 99, 250405 (2007).

[40] O. Gühne, Phys. Rev. Lett. 92, 117903 (2004).

[41] Z. Léka and D. Petz, Probab. Math. Statist. 33, 191 (2013).

[42] D. Virosztek and D. Petz, arXiv:1311.3908.

[43] G. Tóth and D. Petz, Phys. Rev. A 87, 032324 (2013); S. Yu, arXiv:1302.5311.

[44] B. Jungnitsch, T. Moroder, and O. Gühne, Phys. Rev. Lett. 106, 190502 (2011).

[45] T. Moroder and O. Gühne (unpublished). This problem is in the problem book of the National Quantum Information Centre, Gdansk.

[46] M. Navascués, S. Pironio, and A. Acín, Phys. Rev. Lett. 98 , 010401 (2007); New J. Phys. 10, 073013 (2008).

[47] A. C. Doherty et al., Proceedings of IEEE Conference on Computational Complexity 2008 (IEEE, Bellingham, WA, 2008), p. 199.

[48] O. Gühne, Phys. Rev. Lett. 92, 117903 (2004).

[49] O. Gühne, P. Hyllus, O. Gittsovich, and J. Eisert, Phys. Rev. Lett. 99, 130504 (2007).

[50] O. Rudolph, Quantum Inf. Process. 4, 219 (2005); K. Chen and L.-A. Wu, Quantum Inf. Comput. 3, 193 (2003).

[51] G. Tóth and O. Gühne, Phys. Rev. Lett. 102, 170503 (2009); Appl. Phys. B 98, 617 (2010).

[52] This extension is different from the one in observation 2 as it adds new copies of the entire bipartite system, rather than adding new copies of the parties $A$ and $B$. Moreover, it can be shown that since the state $\omega_{12}$ is symmetric, an $1: n$ PPT symmetric extension is the same as an $1+\Delta: n-\Delta$ extension for any integer $0<\Delta<n$.

[53] M. Navascués, M. Owari, and M. B. Plenio, Phys. Rev. A 80, 052306 (2009).
[54] A. Osterloh and J. Siewert, Phys. Rev. A 86, 042302 (2012).

[55] G. Gour, Phys. Rev. A 71, 012318 (2005).

[56] W. Dür, G. Vidal, and J. I. Cirac, Phys. Rev. A 62, 062314 (2000).

[57] The operator $T$ can be obtained based on Eqs. (10) and (11) of Ref. [54].

[58] More precisely, a bound has been developed for the convex roof of $\sqrt{\tau}$ in C. Eltschka and J. Siewert, Sci. Rep. 2, 942 (2012). This quantity is also an entanglement monotone and, for pure states, it remains invariant under determinant 1 stochastic local operations and communication (SLOCC). See also, J. Siewert and C. Eltschka, Phys. Rev. Lett. 108, 230502 (2012).

[59] L. Vandenberghe and S. Boyd, SIAM Rev. 38, 49 (1996); We used YALMIP and SeDuMi. See, J. Löfberg, in Proceedings of the CACSD Conference (Taipei, Taiwan, 2004), p. 284; J. F. Sturm, Optimization Methods Software 17, 1105 (2002).

[60] M. M. Sinolecka, K. Zyczkowski, and M. Kus, Acta Phys. Pol. B 33, 2081 (2002).

[61] G. Vidal and R.F. Werner, Phys. Rev. A 65, 032314 (2002).

[62] C. Eltschka and J. Siewert, Phys. Rev. Lett. 111, 100503 (2013).

[63] F. Verstraete, J. Dehaene, and B. De Moor, Phys. Rev. A 68, 012103 (2003).

[64] D. P. DiVincenzo et al., Entanglement of Assistance, in Quantum Computing and Quantum Communications (Springer, Berlin, Heidelberg, 1999), p. 247.

[65] B. Schumacher and M. D. Westmoreland, Phys. Rev. A 56, 131 (1997).

[66] T. J. Osborne and F. Verstraete, Phys. Rev. Lett. 96, 220503 (2006).

[67] C. Helstrom, Quantum Detection and Estimation Theory (Academic Press, New York, 1976); A. Holevo, Probabilistic and Statistical Aspects of Quantum Theory (North-Holland, Amsterdam, 1982); S. L. Braunstein and C. M. Caves, Phys. Rev. Lett. 72, 3439 (1994). 